\title{
CardioTF, a database of deconstructing transcriptional circuits in the heart system
}

\author{
Yisong Zhen ${ }^{\text {Corresp. } 1}$ \\ ${ }^{1}$ State Key Laboratory of Cardiovascular Disease,Fuwai Hospital, National Center for Cardiovascular Diseases, Chinese Academy of Medical Sciences and \\ Peking Union Medical College, Beijing, China \\ Corresponding Author: Yisong Zhen \\ Email address: zhenyisong@fuwaihospital.org
}

Background. Information on cardiovascular gene transcription is fragmented and far behind the present requirements of the systems biology field. To create a comprehensive source of data for cardiovascular gene regulation and to facilitate a deeper understanding of genomic data, the CardioTF database was constructed. The purpose of this database is to collate information on cardiovascular transcription factors (TFs), position weight matrices, and enhancer sequences discovered using the ChIP-seq method. Methods. The Naïve-Bayes algorithm was used to classify literature and identify all PubMed abstracts on cardiovascular development. The natural language learning tool GNAT was then used to identify corresponding gene names embedded within these abstracts. Local Perl scripts were used to integrate and dump data from public databases into the MariaDB management system (MySQL). In-house R scripts were written to analyze and visualize the results. Results. Known cardiovascular TFs from humans and human homologs from fly, Ciona, zebrafish, frog, chicken, and mouse were identified and deposited in the database. Position weight matrices from Jaspar, hPDI, and UniPROBE databases were deposited in the database and can be retrieved using their corresponding TF names. Gene enhancer regions from various sources of ChIP-seq data were deposited into the database and were able to be visualized by graphical output. Besides biocuration, mouse homologs of the 81 core cardiac TFs were selected using a Naïve-Bayes approach and then by intersecting four independent data sources: RNA profiling, expert annotation, PubMed abstracts and phenotype. Discussion. The CardioTF database can be used as a portal to construct transcriptional network of cardiac development. Availability and Implementation. Database URL: http://www.cardiosignal.org/database/cardiotf.html. 
1 CardioTF, a database of deconstructing transcriptional circuits in

2

3

4

5

6 1. State Key Laboratory of Cardiovascular Disease,Fuwai Hospital, National Center for

7 Cardiovascular Diseases Chinese Academy of Medical Sciences and Peking Union

$8 \quad$ Medical College, Beijing, 100037, People's Republic of China

9

10 Corresponding Author:

11 Yisong Zhen

12 Beilishilu, 167, Beijing, 100037 P.R.China

13 Email address: zhenyisong@fuwaihospital.org 


\section{Abstract}

15 Background. Information on cardiovascular gene transcription is fragmented and far behind the 16 present requirements of the systems biology field. To create a comprehensive source of data for

17 cardiovascular gene regulation and to facilitate a deeper understanding of genomic data, the

18 CardioTF database was constructed. The purpose of this database is to collate information on cardiovascular transcription factors (TFs), position weight matrices, and enhancer sequences

20 discovered using the ChIP-seq method.

21 Methods. The Naïve-Bayes algorithm was used to classify literature and identify all PubMed abstracts on cardiovascular development. The natural language learning tool GNAT was then used to identify corresponding gene names embedded within these abstracts. Local Perl scripts were used to integrate and dump data from public databases into the MariaDB management system (MySQL). In-house R scripts were written to analyze and visualize the results.

Results. Known cardiovascular TFs from humans and human homologs from fly, Ciona, zebrafish, frog, chicken, and mouse were identified and deposited in the database. Position weight matrices from Jaspar, hPDI, and UniPROBE databases were deposited in the database and can be retrieved using their corresponding TF names. Gene enhancer regions from various sources of ChIP-seq data were deposited into the database and were able to be visualized by graphical output. Besides biocuration, mouse homologs of the 81 core cardiac TFs were selected using a Naïve-Bayes approach and then by intersecting four independent data sources: RNA profiling, expert annotation, PubMed abstracts and phenotype.

34 Discussion. The CardioTF database can be used as a portal to construct transcriptional network 35 of cardiac development. 
37 http://www.cardiosignal.org/database/cardiotf.html 
38 Introduction

39 Heart disease is a leading cause of morbidity and mortality in both infants and adults $[1,2]$.

40 Insights into the cause of congenital heart diseases (CHDs) have led to the identification of

41 mutations in essential cardiac transcription factors (TFs) [3]. At the opposite end of the temporal

42 spectrum, some cases of adult cardiac disease have been traced to variation in gene regulatory

43 sequences [4]. Thus, knowledge of TFs, their downstream targets, and the regulatory genomic

44 sequences involved in the heart development will enhance our understanding of heart disease.

45 Although the vast amounts of data generated by high-throughput technologies are archived in 46 databases such as ArrayExpress or GEO of NCBI $[5,6]$, they do not contain cohesive knowledge

47 and lack expert annotation. In addition, the field of cardiac development has experienced

48 accelerated growth that can be attributed to the use of various animal models. However, to our

49 present understanding, few efforts have been made to create a database which collects cardiac

50 transcriptional information across species, thereby limiting the benefits from an evolutionary

51 perspective to study heart development.

52 At present, two branching efforts have been made to archive and analyze the data. One is to

53 construct small scale databases, like BloodChIP or CistromeMap, which are dedicated to

54 collecting specific types of data $[7,8]$. The other approach is to establish a number of consortia,

55 like ENCODE, modENCODE, and Epigenomics Roadmap, which are created to generate huge

56 amounts of raw data and archive them [9-11]. In addition to these projects, analysis and

57 visualization software are valuable resources that lead to deeper understanding of the data, and

58 facilitate the generation of novel hypotheses. Central databases, like Ensembl and UCSC also

59 have search functions which allow browsing of the results generated by the consortia mentioned 
60 above $[12,13]$. However, there are currently few databases committed exclusively to

61 cardiovascular development [14]. This prompted us to combine information about TFs, position

62 weight matrices (PWMs), and ChIP-seq results and create a one-stop site for information on

63 cardiovascular development, thus facilitating systems biology studies in transcriptional network

64 regulation [15].

65 CardioTF was therefore constructed to capture all transcriptional information relating to 66 cardiovascular development. As a biocuration project, it documents TFs, PWM files and 67 enhancers across species, including fly, Ciona, fish, frog, chicken, mouse and human. It also 68 implements a search engine to query this information on the fly. In addition to the data-mining 69 effort, core cardiac TFs are identified using Naïve-Bayes approach, which can be used as a 70 roadmap alongside with further annotation for enhancers to generate gene regulatory network of 71 heart development

\section{Materials \& Methods}

\section{The project's code and data for reproducible research}

74 All the Perl scripts and R codes were uploaded to GitHub (https://github.com/zhenyisong/). The 75 raw data including Weinstein meeting abstracts (positive_test_data plus positive_training_data), 76 negative dataset (negative_test_data plus negative_training_data) (in zip format), intermediary

77 files, which contain cross-validation results as well as other public data were uploaded to the 78 CardioTF database server (http://www.cardiosignal.org/download/download.html). These raw 79 data and source codes can be used to verify the findings.

\section{Comprehensive collection and annotation of cardiac TFs}


81 Cardiac TFs were previously defined as regulators of cardiac gene expression, which can impact

82 the process of heart development, particularly the initiation and maintenance of the myocardium

83 [16]. In the CardioSignal database, efforts were also made to collate the cardiac specific

84 enhancers which drive gene expression in cardiomyocytes. At that time, cardiac specific

85 transcriptional factors were defined as genes that regulated expression of genes in the

86 myocardium. During development, the heart consists of three layers: the myocardium,

87 epicardium and endocardium [17,18]. Additionally, at least four heart-specific cell lineages

88 have been characterized, including cardiomyocytes, endothelial cells, epicardial cells, and

89 fibroblasts, the latter is derived mainly from epicardial cells through the epithelial to

90 mesenchymal transition (EMT) [19,20]. By definition, cardiac TFs themselves should be

91 involved in the steps of specification, determination, patterning, and differentiation that will

92 result in a heart fate. In our CardioTF database we collated, cardiac transcription factors which

93 are expressed in all layers of heart. The goal of the CardioSignal database was to use a machine

94 learning approach to find cardiac enhancers at the genome scale. In contrast, the CardioTF

95 database is constructed to study systems biology of transcription regulation. The cross-talk

96 between different layers will also be explored using this platform. In the initial screen, we

97 identified human TFs from previously published annotations [21], hence it was named

98 Wingender's annotation set. This dataset is comprehensive in annotating human TFs. We used

99 these human TFs, excluding human-specific TFs, as a reference to search for their homologs in

100 other species, including fly, Ciona, zebrafish, chicken, frog and mouse [22]. Human-specific

101 TFs are defined as genes which have no homologs in the mouse genome. The NCBI

102 HomoloGene database [23] was used as a reference to assess homologs between human and

103 mouse/zebrafish. Human homologs from other were retrieved from their central databases, 
104 namely, FlyBase (Fly), BirdBase (Chicken), Aniseed (Ciona) and Xenbase (Frog) [24-27]. We

105 also documented the expression status for mouse TFs from four independent sources which

106 included annotation from the Cardiovascular Gene Ontology Annotation Initiative [28], Mouse

107 Genome Database (MGI) genes with cardiovascular phenotypes [29], PubMed abstract parsing

108 results and RNA expression profiling results.

109 TFs from PubMed abstract parsing

110 The Weinstein Cardiovascular Conference provides a platform for talks and posters on all

111 aspects of heart development and congenital heart disease. The Weinstein meeting abstracts

112 were extracted from the meeting abstract book from 2010 to 2013 by hand. As the positive

113 group, this data set included 954 abstracts. We assumed that Weinstein-like abstracts deposited

114 in PubMed are all from the cardiovascular community and focus on cardiovascular development.

115 Abstracts from the negative group were from non-heart related journals, which were manually

116 selected from the PMC Open Access Subset at NCBI. To choose the negative control journals,

117 the following criteria were set. First, well-known cardiovascular journals were excluded, such as

118 'Circulation' and 'Circulation Research'. Second, journals without key words 'heart' or 'cardiac' 119 or 'cardiovascular' in their title were selected. Third, journals which are dedicated to the study

120 of other organs or diseases, for example, 'Neuron' or 'Cancer' were selected. Fourth, other

121 journal which are unlikely to publish articles about cardiovascular development and related

122 topics, such as journals about plants or viruses, were selected. Journals in the negative group 123 contained research from across kingdoms and topics obviously in other fields, such as 124 'Sleep_Disord' or 'Toxicology'. All journal names in the negative group were saved in a file 125 and uploaded onto the cardioTF server (negative_set.journal.txt). The negative group includes 12657080 abstracts. We split the data (positive and negative groups) into a training (80\%) and test 
127 (20\%) set. The Naïve-Bayes module from The Comprehensive Perl Archive Network (CPAN)

128 was used with a local Perl script to classify Weinstein-like abstracts. We used the training set

129 and adopted the $5 \times 2$ cross-validation proposed by Dietterich [30] to train and validate the data.

130 The parameter (the cutoff to decide whether an abstract is a true Weinstein-like abstract) was

131 selected based on average predictive performance which resulted in a classification accuracy

132 (ACC) of 0.99. A wrapper function was implemented to parse the abstracts and calculate the 133 word frequencies. This function called two Perl modules (Lingua::EN::Splitter and 134 Lingua::EN::StopWords) to extract words and perform text analysis. The word frequency alone 135 was forwarded to the algorithm. The withheld test set using the optimized parameter was then 136 used to assess the algorithm's final performance. All publication abstracts from 2008 to 2013 137 were downloaded to the local environment and analyzed by the algorithm. We targeted journals 138 which had at least 6 publications classified as Weinstein-like abstracts in the six-year period 139 (annual publication rate is $>=1$ ). Then all abstracts from the targeted journal were downloaded. 140 This process was repeated for all journals that met the criteria. The selected abstracts were then 141 processed by GNAT [31] using its default script (test100.sh) to recognize the mouse gene name. 142 The PMID was recorded when the gene name matched the name in the curated mouse TF set.

\section{RNA expression profiling data procession}

144 Affymetrix data (GSE1479) were processed by R using the MAS5 algorithm which provides a 145 present call for each gene (see the script ExtractAffy.R at Github) Gene expression status was 146 defined as "on" if the gene was expressed in any microarray at selected developmental stages 147 and had a present call. RNA-seq data were re-analyzed using the recommended protocol (all 148 raw data identifiers can be retrieved from supplemental Table S2) [32]. Briefly, pre-processing 149 software (FastQC) was used to estimate the read length of raw data. If read length is above 50bp, 
150 Bowtie2 was used. Otherwise, Bowtie was used. mm10 and hg19 are the genome builds used by

151 UCSC. Index and annotation files for Bowtie2/Bowtie were downloaded from Illumina

152 iGenomes project. Genome sequences from UCSC are repeat-masked with lower-case

153 characters. Any gene with an FPKM value greater than 1 was defined as expressed and this

154 threshold was empirically set although justifiable

155 Depositing PWM files

156 The gene symbol was used as the unique identifier to link the original database ID to our local 157 database primary key. A local Perl script was written to change the format to the TRANSFAC 158 style, which was used by our in-house CardioSignalScan program [16]. PWM files were 159 collected from Jaspar, UniPROBE and hPDI databases [33-35]. Users can retrieve their 160 annotations by directing them to the respective database. All the PWMs from those three 161 sources for each TF were deposited in the database. We currently do not use the program 162 implemented by the Zhang lab [36] to check the similarity of PWMs and reduce the redundancy 163 in the collection of PWM files.

\section{Orthologs of TFs from model systems}

165 NCBI has its own gene orthologs that were identified using unpublished algorithm [37]. TFs 166 from mouse, human and zebrafish are annotated by NCBI Homologene [23]. Frog, chicken and 167 Ciona TF homolog annotations were downloaded from their central databases including 168 Xenbase, BirdBase and ANISEED. Fly TFs, which have counterparts in the human proteome, 169 were annotated by the Inparanoid system [38]. Each TF collected in the database was assigned 170 one treeID on the basis of its human counterpart. The treeID is equivalent to a TF family by the 171 recommendation of TFClass [21].

\section{Enhancer curation: TF-ChIP and Histone-ChIP data processing}


173 Raw ChIP-seq data were recruited based upon two criteria: first, whether the source of tissue or

174 cells is from heart or heart progenitor derived cells; second, the DNA-binding protein for the

175 ChIP assay should be pan-enhancer markers or heart lineage specific TFs. In the latter case, the

176 core heart TFs were proposed in our screening procedure. Enhancer regions were defined by

177 ChIP-seq signals. We assume that pan-enhancer markers, like H3K4me1 or H3K27ac [39], or

178 lineage specific markers, like GATA4 or MEF2C [40] will delineate true enhancer regions, 179 although these collections will produce some false positive records. Peak calling was performed 180 using the recommended pipeline [41]. In brief, sequencing reads were aligned to the $181 \mathrm{~mm} 10 / \mathrm{hg} 19$ reference genome using Bowtie/Bowtie2. Mm10/hg19 represents the genome build 182 assigned by UCSC. Index files for mm10/hg19 were downloaded from the iGenome project. 183 MACS1.4.2 was used to process all the ChIP-seq data. The default cutoff for the p-value was 184 1e-05. This default value was used in all ChIP-seq analysis. This protocol was adapted from 185 published literature [42].

186 Bowtie call

187 bowtie -m $2-\mathrm{S}-\mathrm{q}-\mathrm{p} 8$

188 Peak calling was performed using the MACS peak calling algorithm.

189 MACS call linux command

190 macs14 -t ERR231646.bam -c ERR231653.bam -g mm -n sham_Anti_H3k9ac

191 A Torque job script was written to submit the job to the supercomputer. After that, the format 192 transformation was performed:

193 samtools view -bS -o tbx20_positive.bam positive_tbx20.sam

194 When possible, the control files were merged:

195 samtools merge out.bam in_1.bam in_2.bam in_3.bam

196 After MASC analysis was completed, the annotatePeaks.pl was run in HOMER [43] to parse 
197 the bed file from the MACS output. Then the parsed results were dumped into the MySQL table.

198 Public identifiers for the raw data can be retrieved from supplemental Table S2 and ChIP-seq

199 experimental information has been recorded in the MySQL table 'ChIPExpAssay'.

200 Recognition of transcription factor binding sites (TFBSs) in enhancer

201 CardioSignalScan was previously implemented to identify transcription factor binding sites [16].

202 However, this local program (see cardiophylo.pl in GitHub) is brute-force solution which

203 consumes computational time with linear complexity $(\mathrm{O}(\mathrm{mn}))$. In the Big $\mathrm{O}$ notation, $\mathrm{m}$ is the

204 column length of the matrix and $n$ is the length of the input DNA string. Therefore, it is

205 unrealistic to scan sequences longer than 3000bp with this local program. This prompted us to

206 choose MOODS [44] instead, which reduces the computational time proportionally to position

207 weight matrix length $(\mathrm{O}(\mathrm{m}))$. A wrapper module was written to calculate the threshold that

208 gauges the match. The cutoff was empirically defined to be 0.75 (range from 0 to 1 and 1 is

209 most conserved score).

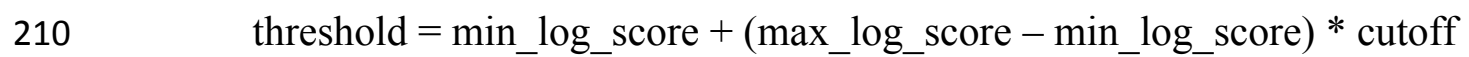

211 This step avoids using $P$-values to assess the significance of TFBS.

\section{Gene ontology analysis}

213 DAVID analysis (version 6.7) was performed using the 81 TFs as the input gene list, official

214 gene symbols as the identifiers and the entire mouse gene set as the background. The functional

215 annotation clusters generated by DAVID were identified by TFs (Figure S2). The classification

216 stringency was set to the default (medium).

\section{Results}

218 The database schema 
219 Our database uses the MariaDB, a drop-in replacement for MySQL, as the database 220 management system (DBMS). To address how information will be stored and how the elements

221 will be related to one another, we used the unified modeling language (UML) to describe the 222 high-level database model [45]. UML was originally developed as a graphical notation for 223 describing software designs in an object-oriented style. It has been extended, and modified and 224 is now a popular notation for describing database designs. Here we used UML instead of an 225 entity/relationship diagram to design the relational database schema following modeling 226 principles, such as faithfulness, avoiding redundancy, and simplicity counts [45] (Fig. 1). Where 227 possible, we used a composition distinguished by a line between two classes that ends in a solid 228 diamond at one end. The diamond implies that the label at the end must be 1:1. For example, 229 there is a composition from CardioTFmatrix to CardioTFCenter, which means that every matrix 230 annotation row (PWM related information) belongs to exactly one row in CardioTFCenter (one 231 type of TF may have more PWM records in a CardioTFmatrix table). A 1:1 label at the 232 CardioTFCenter end is implied by a solid black diamond.

\section{Web Interface and search engine}

234 CardioTF is a Perl website implemented using only Perl language to dynamically display the graphical output while querying the database in the backend (Fig. 2). To aid cardiovascular biologists, a search engine was created to allow users to: (1) identify homology information for

237 the queried TF across six species and link to the corresponding central databases outside 238 CardioTF; (2) identify PWM file union of three public databases regarding the queried TF; and 239 (3) identify the enhancer regions revealed by ChIP-seq data of the queried gene. Thus, the database is able to perform the key functions required to construct a transcriptional network of heart development. 


\section{Cardiovascular TFs in the database}

243 Wingender's annotation set [21] was used as a benchmark to recruit TFs across species. The

244 frozen version of this dataset contains 1564 human TFs. Among them, only 1513 TFs have

245 corresponding Entrez gene records. Human-specific TFs, defined as those with no orthologs in

246 the mouse genome, were discarded because no model system could be used to verify their

247 function in vivo. This step excluded a further 313 human TFs which have no counterpart in

248 mouse from the homolog annotation. Therefore, 1200 mouse TFs were collected. Other

249 established animal models for cardiovascular development include fly, Ciona, zebrafish, frog,

250 and chicken. TFs from these species were collected if they were homologs to the above mouse

251 TFs. The distribution of TFs from different species is shown in Fig. 3. The expression status of

252 mouse TFs was verified by four independent resources, namely RNA-seq data re-analysis [39],

253 phenotype annotations from the MGI database [29], expert recommendation from the UK

254 Cardiovascular Gene Annotation Initiative [28], and PubMed relevance from classification of

255 Weinstein-like abstracts (see the subsequent section and Table S1).

\section{Weinstein TFs from PubMed analysis}

257 We identified the journals that favored Weinstein-style papers, which were likely contain

258 information on genes involved in cardiovascular development. As expected, after using a Naïve-

259 Bayes method, the journals we identified were among the 30 journals most relevant to 260 developmental biology. Two of the journals (Circ. Res. and J Mol Cell Cardiol.) obviously 261 publish research specifically in the area of heart system. (Table S1: CardioJournalDistribution).

262 If normalized and ranked by publication rate, the above conclusion still holds true although two 263 different heart journals (Eur J Echocardiogr, Heart Rhythm) are in the top 30 list (Table S1:

264 CardioJournalDistribution_norm) in this case. We then used GNAT, a tool that recognizes gene 
names in the literature, to recover all TFs mentioned in Weinstein-style abstracts because we

assumed that these TFs are studied by researchers in the cardiovascular community (Fig. 4, Fig.

267 S1 and Table S1).

\section{PWM files collected in database}

269 Public databases for PWM files include UniPROBE, Jaspar, and hPDI, and they provide PWM

270 files for TFs. Jaspar PWM files are curated from the published literatures whereas the other two

271 databases generate PWM files from experiments. Our database integrates these three sources,

272 and the TF PWM can be queried on the basis of the TF name. Search results directly link to the

273 original database through the PWM raw database key. The CardioTFmatrix class contains 904

274 records, and these PWM files can be recognized by our local CardioSignalScan program to

275 search for the motifs in genomic regions.

\section{Core cardiovascular TFs}

277 The 1200 mouse TFs were included in the cardiac TF dataset as the entry point to initiate deep 278 annotation. To define a core set of cardiac TFs, we intersected four independent sources of 279 cardiovascular TF collections. Inclusion of the resulting 81 TFs is supported by their expression 280 status, phenotype annotation, expert recommendation and PubMed relevance (Table S1). We 281 also performed DAVID functional analysis, and found that these TFs are particularly enriched 282 in cardiac muscle differentiation (Fig. S2). 10-20\% of these TFs which are enriched in the 283 Annotation Cluster 7 including Gata4,Gata6, Smad7, Nkx2-5, Tbx2, Tbx5, Foxc1, Foxp1, 284 Prox1, Rara ,Rarb, Rxra, Rxrb, Zfpm2. These 14 TFs, are annotated in the DAVID as being involved in cardiac muscle formation. As we know, the heart system includes the endocardium which is a specialized layer derived from endothelial cells. Cluster 8 from our DAVID analysis includes genes expressed in endothelial cells such as Smad5, Smad7, Meis1, Nkx2-5, Tbx20, 
288 Epas1, Foxc1, Foxo1, Hey1, Hand2, Hif1a, Mef2c, Prrx1, Prox1, Srf, Nr2f2, Tcf21, Vezf1,

289 Zfpm2. Is this set of genes the minimum requirement for cardiac development? Indeed, these

290 four sources of supporting evidence indicate that these TFs genes play a key role in heart

291 development. We wanted to determine if these TFs display specific expression patterns in heart

292 development. A heatmap was generated using seven RNA-seq data sets, including samples from

293 embryonic cells, mesoderm cells, cardiac progenitors, nascent cardiomyocytes and adult heart

294 tissue. This heatmap did not reveal any specific patterns (Fig. S3). In adult tissues, these TFs did

295 not exhibit enriched expression in the adult heart. In the case of TFs which are never expressed

296 at any stage of heart development, no specific expression pattern was revealed by the boxplot

297 assay (Fig. S4-S5).

\section{Cardiovascular enhancers collected in this database version}

299 Few enhancers have already been verified by traditional biological experiments, for example, by

300 using transgenic expression of isolated DNA fragments in vivo to analyze temporal-spatial

301 patterns. Therefore, the ChIP-seq method provides a high-throughput approach to delineate

302 enhancer regions at the genome scale. A standard protocol was used to identify genome-wide 303 locations of transcription/chromatin factor binding sites or histone modification sites from 304 ChIP-seq data (Fig. S6). The present database houses 511,893 enhancer records, covering 305 different stages of heart development. Searching for a single enhancer also provides a user 306 interface to scan the TFBSs. The binding matrices provided in the list come from the core 307 cardiac TFs. The flat text output file contains the matrix key for the TFs in the database and 308 sorts these hits in the increasing order.

\section{Discussion}


310 We identified 5442 TFs from six species, and integrated 904 PWM files from three PWM 311 databases. We also collected 511,893 peak fragments for further analysis. The on-the-fly search

312 tool was implemented to match the core cardiac TFBSs in the specified enhancer sequence. Our

313 database provides a framework where users can query homology information for various TFs 314 across species and PWM information corresponding to TFs and enhancers from high-throughput 315 ChIP-seq data. The curation for cardiac enhancers and TFs facilitate future efforts to construct 316 transcription network.

317 The database now contains the six species which are model organisms for studying heart 318 development (Figure 3A). Ciona is an ideal model used to study the early specification step in 319 the Mesp-lineage. Zebrafish is well suited to perform imaging analysis and for performing 320 quantitative study. The chicken is well suited for lineage tracing ex vivo. The mouse provides a 321 well-studied mammalian model and is most similar to humans. The fly is a valuable model for 322 testing new concepts and is easy to study at the genomic level. New models may be added in the 323 future if they have unique advantage over the other models.

324 Previously, we constructed the CardioSignal database which collates cardiac factors driving genes expressed in a tissue-specific or quantitative manner. Most enhancers archived in the database are expressed specifically in the myocardium. CardioTF is a complementary database 327 that accumulates cardiac TFs expressed in the epicardium, endocardium and myocardium. This 328 information including PWMs can be used not only to find the features of enhancers in a 329 machine learning approach (such the left-right patterning of the heart) but also to reconstruct regulatory network in systems biology.

331 We defined a core set of TFs using four independent sources, namely, RNA profiling, expert 332 curation, PubMed abstract parsing and phenotype annotation to support their roles in 
333 cardiovascular development. In RNA-seq analysis, after pre-processing and post-processing of

334 the adult heart data, the count table contained 48995 genes. After filtering genes with FPKM $>1$,

335 there were 20863 genes remaining. Roughly half of these annotated genes were abandoned

336 because their gene expression level was too low. To analyze PubMed abstracts, a Naïve-Bayes

337 approach was used to classify Weinstein-style abstracts and then pick out the TFs embedded in

338 those abstracts. Most journals containing the key words "heart" or "cardiac" or "cardiovascular"

339 focus on heart pathology or physiology instead of development biology. For example, journals

340 such as "Cardiovascular research", "Circulation", and "Circulation research", accept papers

341 related to the adult cardiovascular system. Most papers published in these journals take a more

342 translational approach which is oriented to bench-side work. Our analysis identified two heart

343 related journals (which is obvious from their name) suggesting that the algorithm was successful

344 in finding the wording pattern present in Weinstein abstracts. The results indicate that most

345 developmental biology manuscripts relating to cardiovascular system are sent to specialized

346 journals that focus on development. Top-tier cardiovascular journals are more likely to publish

347 papers describing the adult cardiovascular system. Our text analysis, whether normalized by

348 publication number or not, had a tendency of identifying journals favored by heart

349 developmental biologists and journals that specialized in developmental biology (Table S1).

350 Traditional definitions of heart specific TFs can often be ambiguous and should not include TFs

351 that only regulate cardiac muscle. In the present definition, heart specific TFs must be detected

352 to be expressed in heart tissue which includes the endocardium and epicardium layers. Both

353 DAVID Annotation Cluster 7 and Cluster 8 contain TFs involved in heart muscle or vascular

354 formation. The DAVID analysis result did not reveal any other clusters with genes involved in

355 kidney, liver or the formation of other organs. Even the expression of GATA4 and MEF2C, 
356 which are de facto cardiac TFs, is not restricted to cardiomyocytes. These TFs are expressed in

357 cardiac progenitors at a certain stage of development. The present approach is empirical and

358 proposes a method which uses four independent data sources to identify true cardiac TFs based

359 on their expression, phenotype, community opinion and PubMed abstracts. These 81 core TFs

360 could be used further to support simulation study to infer the significance in future.

361 In general, the set of core cardiac TFs identified by these sources provide a roadmap for systems

362 biology to construct a transcriptional network of heart development. Current approaches by the

363 Sperling group or the Pu group only report three to four TFs based on ChIP-seq data [40,46].

364 Similar approaches by other genome biologists who tried to find cardiac enhancers on a genome

365 scale have been reviewed elsewhere [47, 48]. However, the information generated from these

366 studies is well below our knowledge of these core cardiovascular TFs, which have multiple

367 sources supporting their role in cardiovascular development.

368 We archived 1200 mouse TFs and wanted to determine at what stage of heart development they

369 were expressed. Our preliminary analysis indicates that approximately 200 TFs have no

370 evidence of their expression pattern, phenotype, expert recommendation, and PubMed abstracts.

371 Whether these TF genes are expressed or play roles in heart disease requires further analysis.

372 The database still lacks cell lineage-based expression profiling data, which will quantify the

373 expression level of various TFs and thus construct a 4-D dynamic expression pattern in vivo.

374 This information could be combined with cell lineage-based ChIP-seq data to create a super-

375 resolution of enhancer tomography.

\section{Conclusions}

377 Modern translational medicine rests upon the progressive study of pathways and principles from

378 model organisms such as yeast, fly, fish, and mice to clinical studies in humans. Therefore, we 
379 recruited TFs from six model organisms which are established models for research on 380 cardiovascular development. The identification and collation of these well-annotated homologs

381 from different organisms will enable investigators to better address the complexities of

382 transcriptional network on heart development [48].

383 We hope that in the near future, single-cell sequencing data may provide comprehensive gene

384 expression information with detailed temporal-spatial resolution, thereby providing insight into 385 the transcription networks that contribute to heart development or heart diseases.. CardioTF

386 database try to collate these cis and trans information and take the initial steps in the 387 construction of a comprehensive transcriptional network.

\section{Supplemental Information}

389 Additional file 0: Four independent sources of cardiac TF lists and preferred journals for 390 Weinstein-like papers. Format: XLSX. Sheet 1 (CardioJournalDistribution) includes the top 100 391 journals that favor Weinstein-like papers. Sheet 2 (NotHeartTFs) includes all TF names that 392 have never appeared in the four sources of supporting evidence. Sheet 3 (MGI_TFs) is the TF names from MGI database. Sheet 4 (PubMed_TFs) lists TF names from Weinstein-like PubMed 394 abstracts. Sheet 5 (Cardio_GO_UK_TFs) includes TF names from UK Cardio-GO project. Sheet 5 (Cardio_lineage_TFs) includes TF names from RNA-seq or microarray analysis. Sheet

6 (core TFs) defines the core 81 TFs known to be involved in heart development. Sheet 7 397 (CardioJournalDistribution_norm) includes journals ranked by publication rate (normalized 398 version)

Additional file 1: ChIP-seq and RNA-seq raw data collection. Format: XLSX. Sheet 1 (ChIP400 seq data) includes all raw ChIP-seq data information, which are also deposited in MySQL table 'ChIPExpAssay'. The MACS processed data are dumped into MySQL table 'CardioEnhancers'. 
402 Sheet 2 (RNA-seq data) includes all raw RAN-seq information. These data were used to 403 generate the Supplemental Figure S3.

404 Additional file 2: Format: PDF. Figure S1. Confusion matrix and precision-recall curve. By 405 convention, the class label of the minority class is positive (Weinstein abstracts), while the class 406 label of the majority class is negative (non-Weinstein-like abstracts). (A) The confusion matrix 407 for a two-class problem. The first column shows the actual class label of the examples, and the 408 first row presents their predicted class label. In the matrix, TP shows the true positive samples, 409 FP shows the false positive samples, TN shows the true negative samples, and FN shows the 410 false negative samples. (B) The precision-recall curve.

411 Additional file 3: Format: PDF. Figure S2. DAVID analysis of 81 core TFs. The results indicate 412 that these TFs are truly associated with cardiac function by GO term enrichment analysis.

413 Additional file 4: Format: PDF. Figure S3. Heatmap of the 81 core cardiac transcriptional 414 factors at the different stages of heart development.

415 Additional file 5: Format: PDF. Figure S4. RNA-seq expression patterns for the 81 core TFs 416 across 13 adult tissues presented by the boxplot.

417 Additional file 6: Format: PDF. Figure S5. The expression profile across 13 adult tissues of TFs 418 which are never expressed in the heart, as determined by our four sources supporting evidence.

419 Additional file 7: Format: PDF. Figure S6. The work flow of parsing ChIP-seq data and 420 dumping it into the MySQL database.

421 Availability and requirements

422 CardioTF database is freely available on the web at 423 http://www.cardiosignal.org/database/cardiotf.html. 


\section{List of abbreviations}

425 TF: transcription factors; PWM: position weight matrix; UML: unified modeling language;

\section{Acknowledgements}

427 The author is grateful to Prof. Rutai Hui, Prof. Weinian Shou, Dr. Tingting Li and Dr. Jianxin

428 Chen for their helpful comments. Special thanks to Prof. Paul Krieg (University of Arizona), 429 Matija Brozovic (ANISEED), Prof. Carl J. Schmidt (BirdBase) and Zichao Sang (CardioSignal) 430 for their comments or suggestions on data curation. I would also like to thank Dr. Rosannah C.

431 Cameron at the Albert Einstein College of Medicine for her assistance editing the manuscript.

\section{References:}

433 1. van der Linde D, Konings EE, Slager MA, Witsenburg M, Helbing WA, Takkenberg JJ, 434 Roos-Hesselink JW..: Birth prevalence of congenital heart disease worldwide: a systematic 435 review and meta-analysis. J Am Coll Cardiol, 2011, 58(21):2241-7.

436 2. Celermajer DS, Chow CK, Marijon E, Anstey NM, Woo KS: Cardiovascular disease in the 437 developing world: prevalences, patterns, and the potential of early disease detection. $J A m$ 438 Coll Cardiol, 2012, 60(14):1207-16.

439 3. McCulley DJ, Black BL: Transcription factor pathways and congenital heart disease. 440 Curr Top Dev Biol, 2012, 100:253-77.

441 4. Smith JG, Newton-Cheh C: Genome-wide association studies of late-onset cardiovascular 442 disease. J Mol Cell Cardiol, 2015, 83:131-41.

443 5. Parkinson H, Sarkans U, Kolesnikov N, Abeygunawardena N, Burdett T, Dylag M, Emam I, 444 Farne A, Hastings E, Holloway E, Kurbatova N, Lukk M, Malone J, Mani R, Pilicheva E, 445 Rustici G, Sharma A, Williams E, Adamusiak T, Brandizi M, Sklyar N, Brazma A..: 
ArrayExpress update--an archive of microarray and high-throughput sequencing-based

447

448

449

450

451

452

453

454

455

456

457

458

459

460

461

462

463

464

465

466

467

468

functional genomics experiments. Nucleic Acids Res, 2011,39(Database issue):D1002-4.

6. Barrett T, Wilhite SE, Ledoux P, Evangelista C, Kim IF, Tomashevsky M, Marshall KA, Phillippy KH, Sherman PM, Holko M, Yefanov A, Lee H, Zhang N,Robertson CL, Serova N, Davis S, Soboleva A..: NCBI GEO: archive for functional genomics data sets--update. Nucleic Acids Res, 2013, 41 (Database issue):D991-5.

7. Chacon D, Beck D, Perera D, Wong JW, Pimanda JE: BloodChIP: a database of comparative genome-wide transcription factor binding profiles in human blood cells. Nucleic Acids Res, 2014, 42(Database issue):D172-7.

8. Qin B, Zhou M, Ge Y, Taing L, Liu T, Wang Q, Wang S, Chen J, Shen L, Duan X, Hu S, Li W, Long H, Zhang Y, Liu XS..: CistromeMap: a knowledgebase and web server for ChIPSeq and DNase-Seq studies in mouse and human. Bioinformatics, 2012, 28 (10):1411-12.

9. Harrow J, Frankish A, Gonzalez JM, Tapanari E, Diekhans M, Kokocinski F, Aken BL, Barrell D, Zadissa A, Searle S, Barnes I, Bignell A, Boychenko V, Hunt T, Kay M, Mukherjee G, Rajan J, Despacio-Reyes G, Saunders G, Steward C, Harte R, Lin M, Howald C, Tanzer A, Derrien T, Chrast J, Walters N, Balasubramanian S, Pei B, Tress M, Rodriguez JM, Ezkurdia I, van Baren J, Brent M, Haussler D, Kellis M,Valencia A, Reymond A, Gerstein M, Guigó R, Hubbard TJ. GENCODE: the reference human genome annotation for The ENCODE Project. Genome Res., 2012, 22(9):1760-74.

10. Celniker SE, Dillon LA, Gerstein MB, Gunsalus KC, Henikoff S, Karpen GH, Kellis M, Lai EC, Lieb JD, MacAlpine DM, Micklem G, Piano F, Snyder M, Stein L, White KP, Waterston RH; modENCODE Consortium. Unlocking the secrets of the genome. Nature., 2009, 459(7249):927-30 
469 11. Romanoski CE, Glass CK, Stunnenberg HG, Wilson L, Almouzni G. Epigenomics:

470 Roadmap for regulation. Nature. 2015, 518(7539):314-6.

471 12. Mangan ME, Williams JM, Kuhn RM, Lathe WC 3rd. The UCSC Genome Browser: What

472 Every Molecular Biologist Should Know. Curr Protoc Mol Biol., 2014, 1;107:19.9.1-19.9.36.

473 13. Flicek P, Ahmed I, Amode MR, Barrell D, Beal K, Brent S, Carvalho-Silva D, Clapham P, 474 Coates G, Fairley S, Fitzgerald S, Gil L, García-Girón C, Gordon L, Hourlier T, Hunt S, 475 Juettemann T, Kähäri AK, Keenan S, Komorowska M, Kulesha E, Longden I, Maurel T, 476 McLaren WM, Muffato M, Nag R, Overduin B, Pignatelli M, Pritchard B, Pritchard E, Riat HS, 477 Ritchie GR, Ruffier M, Schuster M, Sheppard D, Sobral D, Taylor K, Thormann A, Trevanion S, 478 White S, Wilder SP, Aken BL, Birney E, Cunningham F, Dunham I, Harrow J, Herrero J, 479 Hubbard TJ, Johnson N, Kinsella R, Parker A, Spudich G, Yates A, Zadissa A, Searle SM. 480 Ensembl 2013. Nucleic Acids Res., 2013, 41(Database issue):D48-55.

481 14. Djordjevic D, Yang A, Zadoorian A, Rungrugeecharoen K, Ho JW. How difficult is 482 inference of mammalian causal gene regulatory networks? PLoS One., 2014, 9(11):e111661. 483 15. Blais A, Dynlacht BD. Constructing transcriptional regulatory networks. Genes Dev. , $484 \quad 2005,19(13): 1499-1511$.

485 486 487 488 489 490
16. Zhen Y, Wang Y, Zhang W, Zhou C, Hui R. CardioSignal: a database of transcriptional regulation in cardiac development and hypertrophy. Int J Cardiol., 2007, 116(3):338-347.

17. Moorman AF, Christoffels VM. Cardiac chamber formation: development, genes, and evolution. Physiol Rev., 2003, 83(4):1223-1267.

18. Fishman MC, Chien KR. Fashioning the vertebrate heart: earliest embryonic decisions. Development. , 1997, 124(11):2099-2117. 
491 19. Evans SM, Yelon D, Conlon FL, Kirby ML. Myocardial lineage development. Circ Res,. 492 2010,107(12):1428-1444.

493 20. Moore-Morris T, Cattaneo P, Puceat M, Evans SM. Origins of cardiac fibroblasts. J Mol 494 Cell Cardiol., 2016, 91:1-5.

495 21. Wingender E, Schoeps T, Dönitz J: TFClass: an expandable hierarchical classification of 496 human transcription factors. Nucleic Acids Res, 2013, 41 (Database issue):D165-70.

497 22. Hutson MR, Kirby ML: Model systems for the study of heart development and disease. 498 Semin Cell Dev Biol, 2007, 18(1):1-2.

499 23. NCBI Resource Coordinators. Database resources of the National Center for 500 Biotechnology Information. Nucleic Acids Res. 2015, 43(Database issue):D6-17.

501 24. Attrill H, Falls K, Goodman JL, Millburn GH, Antonazzo G, Rey AJ, Marygold SJ; FlyBase 502 consortium. FlyBase: establishing a Gene Group resource for Drosophila melanogaster. 503 Nucleic Acids Res. 2016, 44(D1):D786-92.

504 25. Karpinka JB, Fortriede JD, Burns KA, James-Zorn C, Ponferrada VG, Lee J, Karimi K, 505 Zorn AM, Vize PD.: Xenbase, the Xenopus model organism database; new virtualized 506 system, data types and genomes. Nucleic Acids Res, 2015, 43 (Database issue):D756-63.

507 26. Schmidt CJ, Romanov M, Ryder O, Magrini V, Hickenbotham M, Glasscock J, McGrath 508 S, Mardis E, Stein LD.: Gallus GBrowse: a unified genomic database for the chicken. 509 Nucleic Acids Res, 2008, 36 (Database issue):D719-23.

510 27. Tassy O, Dauga D, Daian F, Sobral D, Robin F, Khoueiry P, Salgado D, Fox V,Caillol D, 511 Schiappa R, Laporte B, Rios A, Luxardi G, Kusakabe T, Joly JS, Darras S, Christiaen L, 512 Contensin M, Auger H, Lamy C, Hudson C, Rothb?cher U, Gilchrist MJ, Makabe KW, Hotta K, 513 Fujiwara S, Satoh N, Satou Y, Lemaire P..: The ANISEED database: digital representation, 
514 formalization, and elucidation of a chordate developmental program. Genome Res, 2010, $515 \quad 20(10): 1459-68$.

516 28. Khodiyar VK, Hill DP, Howe D, Berardini TZ, Tweedie S, Talmud PJ, Breckenridge R,

517 Bhattarcharya S, Riley P, Scambler P, Lovering RC.: The representation of heart 518 development in the gene ontology. Dev Biol, 2011, 354(1):9-17.

519 29. Blake JA, Bult CJ, Eppig JT, Kadin JA, Richardson JE: Mouse Genome Database Group.

520 The Mouse Genome Database: integration of and access to knowledge about the 521 laboratory mouse. Nucleic Acids Res, 2014, 42 (Database issue):D810-17.

522 30. Dietterich TG: Approximate statistical tests for comparing supervised classification 523 learning algorithms. Neural Computation, 1998, 10:1895-923.

524 31. Hakenberg J, Plake C, Leaman R, Schroeder M, Gonzalez G: Inter-species normalization 525 of gene mentions with GNAT. Bioinformatics, 2008, 24(16):126-32.

526 32. Trapnell C, Roberts A, Goff L, Pertea G, Kim D, Kelley DR, Pimentel H,Salzberg SL, Rinn 527 JL, Pachter L.: Differential gene and transcript expression analysis of RNA-seq 528 experiments with TopHat and Cufflinks. Nat Protoc, 2012, 7(3):562-78.

529 33. Mathelier A, Zhao X, Zhang AW, Parcy F, Worsley-Hunt R, Arenillas DJ, Buchman S, 530 Chen CY, Chou A, Ienasescu H, Lim J, Shyr C, Tan G, Zhou M, Lenhard B,Sandelin A, 531 Wasserman WW..: JASPAR 2014: an extensively expanded and updated open-access 532 database of transcription factor binding profiles. Nucleic Acids Res, 2014, 42 (Database 533 issue):D142-7.

534 34. Hume MA, Barrera LA, Gisselbrecht SS, Bulyk ML: UniPROBE, update 2015: new tools 535 and content for the online database of protein-binding microarray data on protein-DNA 536 interactions. Nucleic Acids Res, 2015, 43(Database issue):D117-22. 
537 35. Xie Z, Hu S, Blackshaw S, Zhu H, Qian J: hPDI: a database of experimental human

538 protein-DNA interactions. Bioinformatics, 2010, 26(2):287-9.

539 36. Schones DE, Sumazin P, Zhang MQ. Similarity of position frequency matrices for

540 transcription factor binding sites. Bioinformatics., 2005, 21(3):307-13.

541 37. Altenhoff AM, Dessimoz C: Phylogenetic and functional assessment of orthologs

542 inference projects and methods. PLoS Comput Biol, 2009, 5(1): e1000262.

543 38. Sonnhammer EL, Östlund G: InParanoid 8: orthology analysis between 273 proteomes,

544 mostly eukaryotic. Nucleic Acids Res, 2015, 43 (Database issue):D234-39.

545 39. Shen Y, Yue F, McCleary DF, Ye Z, Edsall L, Kuan S, Wagner U, Dixon J, Lee L,

546 Lobanenkov VV, Ren B. A map of the cis-regulatory sequences in the mouse genome.

547 Nature., 2012, 488(7409):116-20.

548 40. He A, Kong SW, Ma Q, Pu WT: Co-occupancy by multiple cardiac transcription factors

549 identifies transcriptional enhancers active in heart. Proc Natl Acad Sci $U S A$, $550 \quad 2011,108(14): 5632-37$.

551 41. Bailey T, Krajewski P, Ladunga I, Lefebvre C, Li Q, Liu T, Madrigal P, Taslim C, Zhang

552 J.: Practical guidelines for the comprehensive analysis of ChIP-seq data. PLoS Comput Biol, $553 \quad 2013,9(11): \mathrm{e} 1003326$.

554 42. Feng J, Liu T, Qin B, Zhang Y, Liu XS: Identifying ChIP-seq enrichment using MACS. 555 Nat Protoc,2012, 7(9):1728-40.

556 43. Heinz S, Benner C, Spann N, Bertolino E, Lin YC, Laslo P, Cheng JX, Murre C,Singh H,

557 Glass CK: Simple combinations of lineage-determining transcription factors prime cis558 regulatory elements required for macrophage and B cell identities. Mol Cell,2010, $559 \quad 38(4): 576-89$. 
560 44. Korhonen J, Martinmäki P, Pizzi C, Rastas P, Ukkonen E. MOODS: fast search for

561 position weight matrix matches in DNA sequences. Bioinformatics., 2009, 25(23):3181-2.

562 45. Ullman JD, Widom J. A first course in database systems, 3rd. Beijing. Pearson Education 563 Asia Limited \& China Machine Press; 2008. p.140-147.

564 46. Schlesinger J, Schueler M, Grunert M, Fischer JJ, Zhang Q, Krueger T, Lange M,Tönjes M, 565 Dunkel I, Sperling SR: The cardiac transcription network modulated by Gata4, Mef2a, 566 Nkx2.5, Srf, histone modifications, and microRNAs. PLoS Genet, 2011,7(2):e1001313.

567 47. Wamstad JA, Alexander JM, Truty RM, Shrikumar A, Li F, Eilertson KE, Ding H, Wylie JN, 568 Pico AR, Capra JA, Erwin G, Kattman SJ, Keller GM, Srivastava D, Levine SS, Pollard KS, 569 Holloway AK, Boyer LA, Bruneau BG. Dynamic and coordinated epigenetic regulation of 570 developmental transitions in the cardiac lineage. Cell. 2012, 151(1):206-20.

571 48. Wamstad JA, Wang X, Demuren OO, Boyer LA. Distal enhancers: new insights into heart 572 development and disease. Trends Cell Biol. ,2014, 24(5):294-302. 


\section{Figure 1 (on next page)}

Unified modeling language diagram for the Cardio-TF database design.

The six boxes represent the six major classes, namely CardioTFmatrix, CardioTFCenter, CardioTree, CardioGeneNames, CardioEnhancer, and ChIPExpAssay. These classes are analogous to entity/relationship sets. Each class has two sections, one for the class name and one for the attributes. The attribute of each class is associated with the type used in MariaDB. The "\#" in front of an attribute indicates that it's visibility is "protected", thereby making it a primary key. These classes faithfully represent the real world. 


\begin{tabular}{|l|}
\hline \multicolumn{1}{c|}{ CardirofeneNames } \\
\hline +CardioGeneKey: CHAR( $(9)$ \\
+EntrezGeneID: INT,UNSIGNED \\
+GeneName: VARCHAR(80) \\
\hline
\end{tabular}

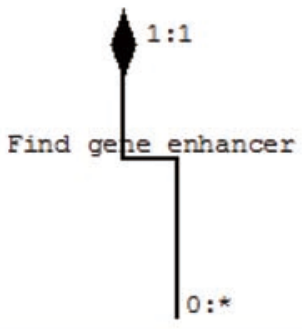

CardioEnhancer

-EnhancerKey. CHAR(20)

+enhancerIntKey. INT, UNSIG NED

+CardioGeneKey. CHAR(9)

+GenomeVersion CHAR(5)

+PositionStart INT UNSIGNED

+PositionEnd: INT UNSIGNED

+L ength INT UNSIGNED

+OriginalID: VARCHAR(30)

+Peakscore: FLOAT UNSIGNED

+Genom eposition: VARCHAR(120)

+ChIPExpID: CHAR(5)
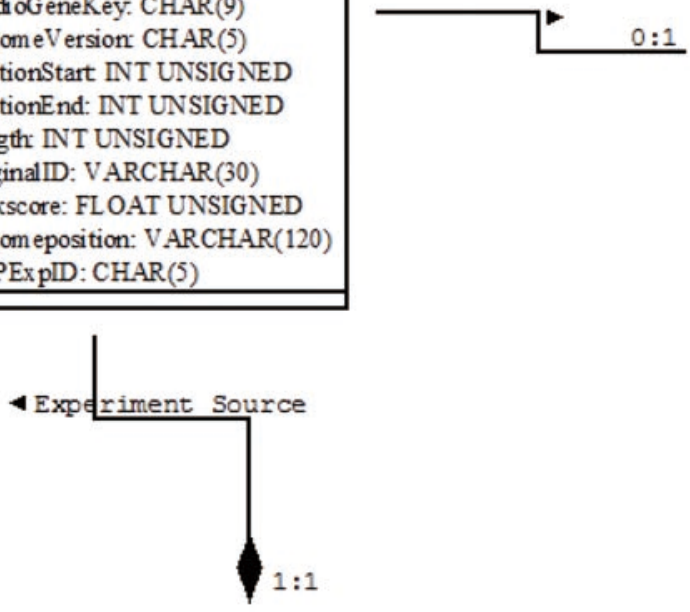

$1: 1$

$0: 1$

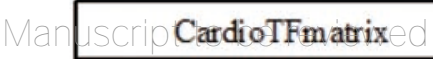

\#CardioMatrixKey. CHAR(7)

+Car dioMatrixInt INT UNSIGNED

+CardioIfmatrix: Text

+ DB source: $E N U M$

+DBforeignKey. VARCHAR(20)

+CardioTFInt INT UNSIGNED

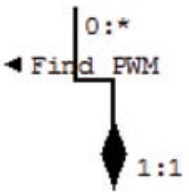

$1: 1$

\begin{tabular}{|l|}
\hline \multicolumn{1}{|c|}{ CardioTFCenter } \\
\hline \#-CardioTFKey: CHAR(7) \\
+CardioTFInt INT UNSIGNED \\
+EntrezGeneID: INT \\
+SwissProID: CHAR(6) \\
+HUGO Gene Symbol: VARCHAR(80) \\
+-SpieceID: ENUM \\
+DBForeignKey: VARCHAR(40) \\
+DBname: ENUM \\
+HomologGeneCardID: VARCHAR(20) \\
+treeID: CHAR(5) \\
\hline
\end{tabular}

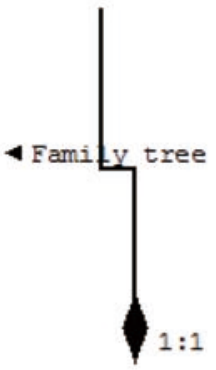

ChIPExpAssay

=ChIPExpID: CHAR(5)

+PMID: INT,UNSIGNED

+ChIPAntibody: VARCHAR(20)

+AntibodyBrand: VARCHAR(20)

+C ellSource: VARCHAR(50)

\section{CardioTFtree}

\#treeIDKey: CHAR(5)

+treeIDInt SMALLINT UNSIGNED

+treeSuperClass: VARCHAR(80)

+treeClass: VARCHAR(80)

treeF amily: V ARCHAR(80) 


\section{Figure 2 (on next page)}

The search engine and the web interface of the database.

(A) The search engine was implemented to perform three functions: querying TFs, their PWMs and gene enhancers (B) Web graphical output of Gata4 enhancers in mouse. Black lines indicate the enhancer regions found by the ChIP-seq scanning program. These regions are from the same specie, but might be from different experiments. A user can check the experiment inforamtion by clicking the E0000XXX link which represents the primary key (ID) for this enhancer region, thus allowing the user to save it and retrieve the information later. (C) Query results for the GATA4 TF across species. TFs are listed and indexed according to their database identifiers. 
A PoerJ

A CardioTF Module: Cardiac Transcriptional Factor (TF) V OPTIONS: Cardiac Gene Name

QUERY: Gata4

\section{submit}

B

gata4 Enhancer search result

Chr. coordinate 63221298 (start)

63331906 (end)

chromosone

E00060371

E00239996

E00285759

E00186993

E00131292

E00001403

E00065127

E00285717

$E 00003078$

E00239682

E00130653

E00187482



(1)

C

\begin{tabular}{|c|c|c|c|c|c|}
\hline CardioTF Identifier & Gene Symbol & Species Name & EntrezGenerD & SwissProID & DB_link \\
\hline TF02887 & gata4 & Danio rerio & 30483 & B8JKU1 & ZFIN \\
\hline TF01887 & GATA4 & Homo sapiens & 2626 & P43694 & NCBI \\
\hline TF04086 & gata4 & Xenopus (Silurana) tropicalis & 549703 & Unknown & XenBase \\
\hline TF04714 & GATA4 & Gallus gallus & 396392 & Unknown & BirdBase \\
\hline TF05400 & PNR & Drosophila melanogaster & 44849 & P52168 & FlyBase \\
\hline TF01888 & 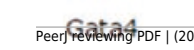 & 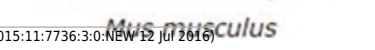 & 14463 & Q08369 & MGI \\
\hline
\end{tabular}


Figure 3 (on next page)

TF distribution across species in the database.

(A) Phylogenetic tree showing the main animal models commonly used in heart development research and their evolutionary relationship. The divergence times in millions of years ago (Mya) are shown on the basis of multigene and multiprotein studies. Branch lengths are not proportional to time (B) Distribution of TFs across six species. All TFs have homologs in humans. The unit of Y-axis is TF number. 
$A_{\text {PeerJ }}$
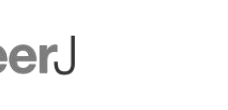

Homo sapiens

Manuscript to bareviewed

Gallus gallus

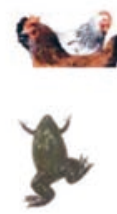

990

460

Xenopus

Danio rerio

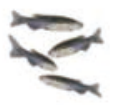

Ciona intestinalis

Drosophila melanogaster
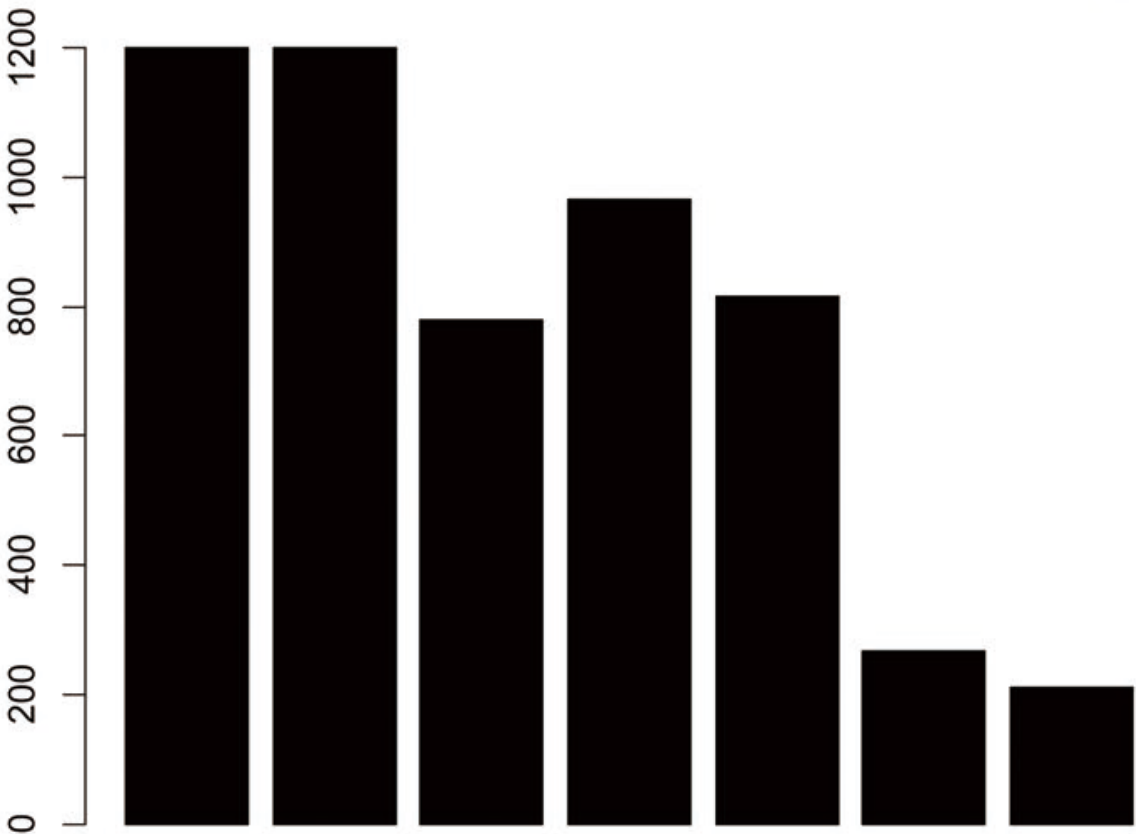

Peer) reviewing PDF | (2015:11:7736:3:0:NEW 12 Jul 2016) 


\section{Figure 4(on next page)}

Machine learning protocol used to select TFs described in Weinstein-like papers.

(A) The pipeline used to select TF gene symbols from Weinstein PubMed abstracts. First, a Naïve-Bayes module was used to select Weinstein-like papers from PubMed abstracts. Second, GNAT, a software that recognizes gene symbols, was used to identify all TF names from these Weinstein-like papers. (B) ROC curve and prediction performance judged by sensitivity, precision and F1 score. 
A
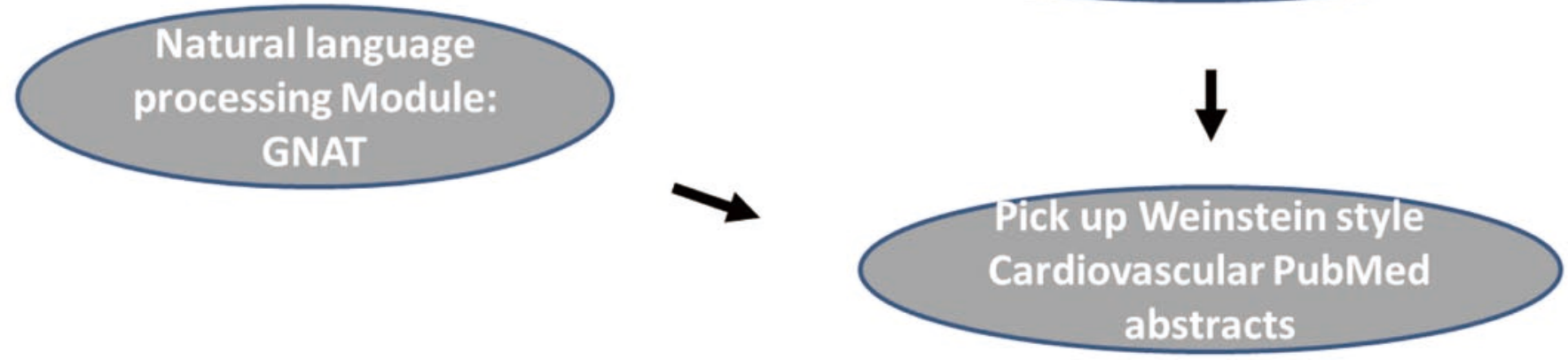

B

\section{Gene symbol/Entrez GenelD}

\section{Sensitivity $\quad 0.9316$}

\section{Precision $\quad 0.9516$}

\section{F1 Score $\quad 0.9415$}

